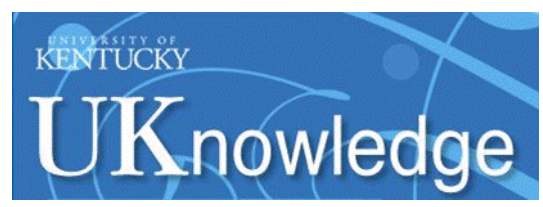

University of Kentucky

UKnowledge

\title{
Impact of Home Visit Capacity on Genetic Association Studies of Late-Onset Alzheimer's Disease
}

\author{
David W. Fardo \\ University of Kentucky, david.fardo@uky.edu \\ Laura E. Gibbons \\ University of Washington \\ Shubhabrata Mukherjee \\ University of Washington \\ M. Maria Glymour \\ University of California - San Francisco \\ Wayne McCormick \\ University of Washington

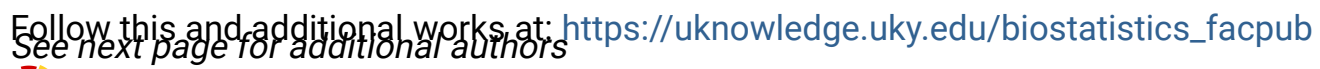 \\ Part of the Biostatistics Commons, Demography, Population, and Ecology Commons, Diseases \\ Commons, and the Genetics and Genomics Commons \\ Right click to open a feedback form in a new tab to let us know how this document benefits you.
}

\section{Repository Citation}

Fardo, David W.; Gibbons, Laura E.; Mukherjee, Shubhabrata; Glymour, M. Maria; McCormick, Wayne; McCurry, Susan M.; Bowen, James D.; Larson, Eric B.; and Crane, Paul K., "Impact of Home Visit Capacity on Genetic Association Studies of Late-Onset Alzheimer's Disease" (2017). Biostatistics Faculty Publications. 41.

https://uknowledge.uky.edu/biostatistics_facpub/41

This Article is brought to you for free and open access by the Biostatistics at UKnowledge. It has been accepted for inclusion in Biostatistics Faculty Publications by an authorized administrator of UKnowledge. For more information, please contact UKnowledge@lsv.uky.edu. 
Impact of Home Visit Capacity on Genetic Association Studies of Late-Onset Alzheimer's Disease

Digital Object Identifier (DOI)

https://doi.org/10.1016/j.jalz.2017.01.012

Notes/Citation Information

Published in Alzheimer's \& Dementia: Journal of the Alzheimer's Association, v. 13, issue 8, p. 933-939.

(c) 2017 the Alzheimer's Association. Published by Elsevier Inc. All rights reserved.

This manuscript version is made available under the CC-BY-NC-ND 4.0 license

http://creativecommons.org/licenses/by-nc-nd/4.0/.

The document available for download is the author's post-peer-review final draft of the article.

Authors

David W. Fardo, Laura E. Gibbons, Shubhabrata Mukherjee, M. Maria Glymour, Wayne McCormick, Susan M. McCurry, James D. Bowen, Eric B. Larson, and Paul K. Crane 
Published in final edited form as:

Alzheimers Dement. 2017 August ; 13(8): 933-939. doi:10.1016/j.jalz.2017.01.012.

\title{
Impact of home visit capacity on genetic association studies of late-onset Alzheimer's disease
}

\author{
David W. Fardo, $\mathrm{PhD}^{\mathrm{a}}$, Laura E. Gibbons, $\mathrm{PhD}^{\mathrm{b}}$, Shubhabrata Mukherjee, $\mathrm{PhD}^{\mathrm{b}}, \mathrm{M}$. Maria \\ Glymour, $S_{c}{ }^{c}$, Wayne McCormick, MD MPH ${ }^{b}$, Susan M. McCurry, PhD ${ }^{d}$, James D. Bowen, \\ $M^{\mathrm{e}}$, Eric B. Larson, MD MPH ${ }^{f}$, and Paul K. Crane, MD MPH ${ }^{\mathrm{b},{ }^{*}}$ \\ aDepartment of Biostatistics and Sanders-Brown Center on Aging, University of Kentucky, \\ Lexington, $\mathrm{KY}$ \\ bDepartment of Medicine, University of Washington, Seattle, WA \\ 'Department of Epidemiology, University of California at San Francisco \\ dDepartment of Psychosocial and Community Health, University of Washington, Seattle, WA \\ 'Department of Neurology, Swedish Medical Center, Seattle, WA \\ fVice President for Research and Executive Director of the Group Health Research Institute, \\ Group Health, Seattle, WA
}

\section{Abstract \\ INTRODUCTION—Findings for genetic correlates of late onset Alzheimer's disease (LOAD) in studies that rely solely on clinic visits may differ from those with capacity to follow participants unable to attend clinic visits.}

\begin{abstract}
METHODS-We evaluated previously-identified LOAD-risk single nucleotide variants in the prospective Adult Changes in Thought study, comparing hazard ratios (HRs) estimated using the full data set of both in-home and clinic visits $(\mathrm{n}=1697)$ to HRs estimated using only data that was obtained from clinic visits $(\mathrm{n}=1308)$. Models were adjusted for age, sex, principal components to account for ancestry, and additional health indicators.
\end{abstract}

RESULTS—LOAD associations nominally differed for 4 of 21 variants; $C R 1$ and $A P O E$ variants were significant after Bonferroni correction.

DISCUSSION-Estimates of genetic associations may differ for studies limited to clinic-only designs. Home visit capacity should be explored as a possible source of heterogeneity and potential bias in genetic studies.

\footnotetext{
*Corresponding author: Paul K. Crane, MD MPH, Box 359780, 325 Ninth Avenue, Seattle, WA 98104. pcrane@uw.edu. (206) 744-1831 (phone); (206) 897-4688 (fax).

Publisher's Disclaimer: This is a PDF file of an unedited manuscript that has been accepted for publication. As a service to our customers we are providing this early version of the manuscript. The manuscript will undergo copyediting, typesetting, and review of the resulting proof before it is published in its final citable form. Please note that during the production process errors may be discovered which could affect the content, and all legal disclaimers that apply to the journal pertain.
} 


\section{Keywords}

Home research study visits; research clinic study visits; missing data; bias; prospective studies; cohort studies; longitudinal studies; inference; late-onset Alzheimer's disease; genetics; genomewide studies; genome-wide association studies; ascertainment bias; selection bias; populationbased studies

\section{Introduction ${ }^{1}$}

Study design is underemphasized in planning or interpretation of many genome-wide association studies (GWAS) and sequencing projects [1]. In many settings, issues such as sampling, recruitment and data collection strategies are assumed to be of secondary importance, although the potential for bias is well-established. Many GWAS analysis projects amass participants from cohorts with varying recruitment strategies and phenotyping protocols. In resulting manuscripts, these details are often relegated to supplementary information or not described at all.

Even subtle differences in subject ascertainment between studies could produce result heterogeneity, and such heterogeneity may be due to true differences in the relevance of genetic variants across subgroups or due to bias induced by selection processes. Many GWAS statistical models include few covariates, so subgroup effect heterogeneity is not explored and there is little hope of correcting selection bias. If gene-environment interactions exist, or if the genetic effect occurs only in a subgroup of people, success of the GWAS framework may be especially dependent on the sample selection process as it affects the distributions of potentially important subject characteristics. More troubling, if genetic variants and symptoms of incipient $\mathrm{AD}$ interact in determining chances of participating in genetic studies, the observed association between the genetic variant and AD may be severely biased when estimated in the participants. Indeed, such a process can create an observed association that does not match the true effect even among study participants. Of course, most studies cannot evaluate this possibility because they have no information on study non-participants. Not accounting for these scenarios can result in bias and/or diluted statistical power [2-6].

We recently published findings from a prospective cohort with a two-stage sample design indicating that genetic associations with LOAD for the $A P O E$ locus may differ for individuals who attend in-person clinic visits than for the larger community-dwelling population, which includes some people who do not attend study visits in a research clinic[7]. We determined that risk factor associations with LOAD differed for participants who had only in-clinic visits versus the full sample that also included people seen at home. For $A P O E \varepsilon 4$ the estimated hazard ratio for incident LOAD in the full sample was 1.66 (95\% confidence interval [CI] 1.37, 2.01), while in the clinic only dataset, $A P O E \varepsilon 4$ had a hazard ratio of $2.28(95 \% \mathrm{CI} 1.57,3.30)$. The $p$-value for this difference was 0.008 . $A P O E$ was the only genetic factor we evaluated.

\footnotetext{
${ }^{1}$ Abbreviations: ACT: Adult Changes in Thought. AD: Alzheimer's disease. CI: Confidence interval. HR: Hazard ratio. LOAD: Lateonset $\mathrm{AD}$.
} 
The notion that study design could be important in the relevance and magnitude of associations with $A P O E$ has been known previously[8]. Indeed, while $A P O E$ genotype may be strongly predictive of LOAD status in specialty clinic settings, this association is attenuated in community-based settings[9]. We hypothesized that this pattern could be explained by selection bias due to specialty clinic studies lacking home study visit capacity. This phenomenon may also apply to other genetic variants. It is important to distinguish between the consequences of bias (e.g., estimated effects that are not, on average, centered about the true value and diminished power (i.e., less chance of discovering a true effect).

We used genetic and research study data from the Adult Changes in Thought (ACT) study to determine whether home study visit capacity would have an influence on the strengths of association with LOAD found in ACT with LOAD SNVs from the largest LOAD GWAS meta-analysis to date[10].

\section{Methods}

\subsection{Parent study description, ethical considerations, and funding}

Methods for ACT have been published[11-13]. The original cohort enrolled 1994-1996 included 2,581 randomly selected dementia-free people age $\ 65$ who were members of Group Health, a Washington State health care system. An additional 811 participants were enrolled 2000-2003, and in 2005 we began continuous enrollment. Participants are evaluated at 2-year intervals at a research clinic or in their home at the participant's choice. Other than location (i.e., home vs. clinic), screening research study visits are identical.

Study procedures were approved by Institutional Review Boards of Group Health and the University of Washington. Participants provided written informed consent. ACT is supported by the National Institute on Aging, which had no role in study design; in the collection, analysis, and interpretation of data; in the writing of the report; or in the decision to submit the paper for publication.

\subsection{LOAD identification}

Participants were assessed at home or in clinic every 2 years with the Cognitive Abilities Screening Instrument (CASI), for which scores range from 0 to 100 and higher scores indicate better cognitive functioning[14]. Participants with scores of 85 or less underwent further evaluations, including a clinical examination and a battery of neuropsychological tests; dementia evaluations are in the participant's home regardless of the location of the triggering/screening visit. Results of these evaluations, laboratory testing, and imaging records were reviewed in a consensus conference, where research criteria were used to identify cases of dementia[15] and probable or possible AD[16]. Dementia-free participants continued with scheduled follow-up visits. In this study, we are examining whether associations between genetic variants and LOAD differ for people who participated in biennial screening visits in the clinic compared to all study participants; by design in ACT, the dementia evaluations all occur in the participant's home, so the location of these evaluations is not under study here. 


\subsection{Genotyping}

ACT participants were genotyped in two waves. The bulk of the cohort was genotyped using the Illumina Human 660 Quad chip, and a subsequent genotyping wave used the Illumina OmniExpress chip. Data from European Americans from both samples were imputed to the same CEU Human HapMap reference panels as used in the International Genomics of Alzheimer's Project (IGAP) paper from Lambert et al[10]. APOE genotype was obtained separately using standard procedures. Of the other $21 \mathrm{SNVs}$ identified as the top hits in Lambert et al.[10], 20 were available in the ACT data either being directly genotyped or successfully imputed; the lone exception was the DSG2 SNV.

\subsection{Statistical analyses}

We limited our analyses to European Americans based on principal components analysis (a conventional statistical genetics approach to discern ancestry from GWAS data[17]) as well as self-reported white race. We constructed two datasets from the subset of the ACT study with at least one follow-up visit and full genotype data ( 1/3 of ACT participants): a complete data set including information for all participants and all visits (the "all visits" dataset), and a second set that mirrored what a clinic based study would have achieved by excluding people who never attended a clinic visit (the "clinic only" dataset). By definition, participants whose entire set of visits consisted of home visits are not included in the "clinic only" dataset. The "all visits" dataset comprises 1697 individuals with a total of 7997 follow-up visits, 15,972 person-years of time in the ACT study, and 419 cases of incident dementia or LOAD resulting in an incidence rate of 26.2 cases per person-year. The "clinic only" dataset includes 1308 individuals with a total of 5106 follow-up visits, 10,350 personyears, and 96 cases of incident dementia or LOAD corresponding to an incidence rate of 9.3 cases per person-year.

We modeled probable or possible AD as the dependent outcome[16]. Because the ACT study is a prospective cohort study and all cases were incident cases of LOAD, we used Cox proportional hazards regression models with age as the time axis[18] and included age at baseline, sex, and three principal components as covariates. For our primary analyses, we also included years of formal schooling as well as self-reports from baseline of diabetes, hypertension, coronary heart disease (a composite including self-reported heart attacks, angina, coronary artery bypass grafting, or angioplasty), cerebrovascular disease (a composite including stroke, transient ischemic attack, or carotid surgery), and quartiles of medical comorbidity as estimated with the prescription-medications-based RxRisk measure[19]. The assumption of proportional hazards was tenable for all models. We also evaluated logistic regression models including only sex, age at baseline, and three principal components to facilitate a more direct comparison with the Lambert et al. analyses[10]. Our primary analyses with multiple covariates are analogous to epidemiological investigation of candidate risk factors, while our sensitivity analyses are analogous to standard genome-wide discovery analyses in GWASs.

We compared hazard ratios from the "all visits" and "clinic only" datasets using a bootstrapping procedure to evaluate whether differences were plausibly due to sampling variability (i.e., chance). We drew (with replacement) random subsets from the "all visits" 
dataset that were the same size as the "clinic only" dataset. Each randomly drawn resample has the same size as the "clinic only" dataset, allowing us to evaluate how often, if the "clinic only" dataset was a random sample of the full data set, we would see associations as extreme or more than we observed in the actual "clinic only" dataset. In each resampled "clinic only" dataset, we performed the same adjusted analyses of the association between the SNV and LOAD risk. We determined the proportion of resampled "clinic only" datasets with hazard ratios more extreme than those from the actual "clinic only" dataset. The bootstrap $p$-values indicate the proportion of drawn datasets with more extreme findings than the actual "clinic only" dataset, which help us understand whether "clinic only" and "all visits" hazard ratios differ more than expected by chance alone. We compared the absolute values of effect sizes in the actual "clinic only" data set to the resampled datasets so we have a 2 -sided $p$-value. The bootstrapped $p$-values represent the evidence of incompatibility with the given statistical model that includes the assumption that the "clinic only" dataset is a random sample from the "all visits" dataset. Thus, they provide a test of whether the differences between the HRs estimated in the "clinic only" and "all visits" datasets could plausibly be due to chance. We consider both nominal $(\mathrm{p}<0.05)$ and Bonferroni corrected $(\mathrm{p}<0.05 / 21)$ thresholds.

We used Stata 14.1 (StataCorp, College Station, TX) for all analyses.

\section{Results}

Demographic and clinical data from the LOAD cases and controls are shown in Tables 1 and

2. These data comprise the "all visits" dataset. There were many distinct patterns of home and clinic visits over time for ACT participants. The distribution of pattern types is presented in Table 2. For the "clinic only" dataset, we excluded all home visit data. This means the 163 people with no clinic visits were excluded. In addition, 389 participants with fewer than two clinic visits were excluded because they had no clinic follow-up to contribute to survival time. In addition, we excluded the home visits (but kept the clinic visits) for 913 participants with a mix of home and clinic visits, usually resulting in shorter follow-up time and sometimes missing a dementia diagnosis triggered by a home visit for those study participants. Minor allele frequencies for variants in genes other than $A P O E$ are in Supplementary Table 1.

In the "all visits" dataset, we observed significant associations with LOAD hazard for SNVs in $C R 1$ (rs6656401, $p=0.007), C D 2 A P(\mathrm{rs} 10948363, p=0.006), P T K 2 B$ (rs28834970, $p=0.009), C D 33$ (rs3865444, $p=0.026), A B C A 7$ (rs4147929, $p=0.023$ ), and $A P O E$ $(p<0.0001)$ (see Table 3$)$. Hazard ratios quantify different measures of risk than odds ratios. Nevertheless, the corresponding HR estimates are of similar magnitude and in the same direction as the OR estimates from Lambert et al.[10] (Table 3 and Supplementary Table). For only two of these loci - CR1 and $A P O E$ - were results significant in the "clinic only" dataset (both $p$-values $<0.0001$ ); no other loci had significant associations with LOAD hazard for the "clinic only" sample.

We next evaluated whether differences in estimates from the "clinic only" dataset and the "all visits" dataset were larger than expected by chance alone. Four of the 21 investigated 
SNVs had nominally significant bootstrap $p$-values; the difference between the HR in the clinic only data set and the HR in the all visit data set was unlikely to have emerged by chance due to sampling variability. In decreasing order of significance, we observed nominally significant results for $A P O E$ ( $p<0.0001), C R 1$ (rs6656401, $p=0.0001$ ), $C D 33$ (rs3865444, $p=0.007$ ), and EPHA1 (rs11771145, $p=0.014$ ). Three of these $-A P O E, C R 1$, and $C D 33$ - were associated with significant LOAD hazard in the "all visits" dataset. When employing logistic regression to calculate effect estimates, odds ratios differed significantly between the clinic only and all visit data sets for these same three variants, plus the PICALM SNV (rs10792832, $p=0.014$ ) (Supplementary Table 2). After Bonferroni adjustment for testing each of $21 \mathrm{SNVs}$, the results from $A P O E$ and $C R 1$ remained significant.

The $A P O E \varepsilon 4$ allele was associated with higher risk of $\mathrm{LOAD}$, with hazard ratio (HR) for the "all visits" dataset of 1.92 (95\% CI 1.60, 2.32). As in our previous publication, the strength of this association was much higher for the "clinic only" dataset, with a HR of 3.26 (95\% CI 2.30, 4.63). These estimates were more divergent than likely to occur by chance alone (bootstrapping $p<0.0001$ ). Similar to $A P O E$, results for $C R 1$ on chromosome 1 (rs6656401) were much stronger in the "clinic only" dataset, with HR 2.13 (95\% CI 1.49, 3.05), than the "all visits" dataset (HR 1.29, 95\% CI 1.07, 1.55). Bootstrapping again confirmed that these estimates were much more distinct than expected by chance alone (bootstrapping $p=0.0001$; Figure 1).

Results for $C D 33$ on chromosome 19 (rs3865444) from the "all visits" dataset (HR 0.84, 95\% CI 0.71, 0.98) were similar to those observed in Lambert et al. (odds ratio=0.94, 95\% CI 0.91, 0.96)[10]. Results for the "clinic only" dataset were in the opposite direction ( $\mathrm{HR}=1.12,95 \% \mathrm{CI} 0.81,1.55)$, though the confidence interval was broad. Bootstrapping results for rs3865444 were nominally significant (bootstrapping $p=0.007$ ). Results for rs11771145 associated with EPHA1 on chromosome 7 were essentially null for the "all visits" dataset ( $\mathrm{HR}=1.02)$, and the confidence interval included the odds ratio estimate from Lambert et al. (OR $=0.9095 \%$ CI 0.88, 1.18[10]). The result for the "clinic only" dataset ( $\mathrm{HR}=0.83$ ) was stronger than that seen in Lambert et al., though the confidence interval was wide and included both the point estimate from the "all visits" dataset and that reported by Lambert et al. ( $\mathrm{OR}=0.9095 \% \mathrm{CI} 0.62,1.13)$. Bootstrapping results for rs11771145 suggested this difference was nominally significant (bootstrap $p=0.014$ ).

For all loci, results from models that included adjustment only for age, sex, and three principal components were similar (not shown).

\section{Discussion}

The ACT study enables each participant to choose whether they would like to have each study visit at a central research clinic or at their own home. As participants age, higher proportions of participants opt for home study visits[7]. In particular, as many participants progress to dementia, their ability or willingness to navigate the world to attend a research clinic visit is dramatically attenuated, as indicated by the fact that of the 419 people with incident LOAD in this report, only 96 had their AD diagnosed following an in-clinic study 
screening visit; the remaining 319 people (76\%) had their LOAD diagnosed following an inhome study screening visit. By design, all detailed dementia diagnosis evaluations occur at participant homes; we focused here on the location of the screening visits that were either in our research clinic or in the participant home. Because the ACT study includes capacity for home study screening visits, we are able to address the question of whether associations from all ACT study screening visits are different from associations observed among the subset of study screening visits at our research clinic. We previously demonstrated large differences for risk factors for LOAD and for associations between neuropathology findings at autopsy and dementia status during life[7].

Here we performed similar analyses but considered associations with SNVs previously found to be associated with LOAD risk[10]. The "clinic only" dataset was smaller than the all-visit cohort in terms of the number of people ( $n=1308$ vs. $n=1697$ ), follow-up time (around 10,000 person-years vs. nearly 16,000 person-years), and number of incident LOAD cases (96 vs. 419). It was thus not a surprise that more loci were nominally statistically significantly associated with LOAD risk in the "all visits" dataset. As in our previous publication, we found large differences between the "clinic only" dataset and the "all visits" dataset for $A P O E$ genotype. $A P O E \varepsilon 4$ alleles were associated with increased risk for LOAD in the "all visits" dataset, with a HR of 1.92 , but they were much more strongly associated in the "clinic only" dataset, with a HR of 3.26. We used bootstrapping to determine the statistical significance of this difference, and found this difference in risk estimates to be very unlikely to be due to chance alone (bootstrapping $p<0.0001$ ). This provides strong evidence against chance being responsible for differences, which leads to a conclusion that the assumptions underlying the model (e.g., no selection bias and no confounding) likely do not hold. We similarly found large differences for rs6656401 associated with $C R 1$, where the HR estimate was much smaller for the "all visits" dataset (1.29) than the "clinic only" dataset (2.13) (bootstrapping $p<0.0001$ ). We found a smaller difference for rs 3865444 associated with $C D 33$, however the estimates were in opposite directions, such that the "all visits" estimate was consistent with the previously published association from Lambert et al. ("all visits" $\mathrm{HR}=0.84$; "clinic only" $\mathrm{HR}=1.12$; bootstrapping $p=0.007$ ).

Thus, with "clinic only" data, we know that the results may be different than what we would observe with a more complete dataset that more closely resembles the underlying population, but we do not know in which direction to expect any differences. Taken together with our prior investigations[7] and viewed as a missing data problem, these results provide further evidence that data missing due to the lack of home study visit capacity are missing not at random (MNAR). This conclusion has important implications. Results from studies with outcomes that are MNAR may be biased - they provide the wrong answer - and we do not know the direction of the possible bias. It is not difficult to envision a scenario such that the act of opting for a home visit relays important information regarding LOAD risk and the subsequent statistical models needed to estimate causal effects.

When data are MNAR, deriving unbiased effect estimates relies on incorporating corrections for the missingness mechanism (i.e., what causes people to select out of clinic-based studies). Since the missingness mechanism is almost never known, this relies on sensitivity 
analyses exploring a range of possible missingness mechanisms [20]. Our results can help guide such sensitivity analyses in data sets that include only clinic-based data.

We chose to focus only on SNVs previously identified to be associated with LOAD risk. Most of these SNVs have been replicated in multiple waves of sampling, though rs 3865444 associated with $C D 33$ was essentially null in the second stage of the Lambert et al. study[10]. Since the Lambert et al. paper includes data from many studies with many study designs, some of which include and others of which do not include home study visit capacity, there may well be additional SNVs that would be discovered if all of the data were from studies that include home visit capacity, but that are not identified with genome-wide significance levels at present. There is additional expense associated with following older study participants at home. Our results strongly support the value of this expenditure in terms of the validity of genetic findings.

A similar point can be made in a much broader context outside of home visit capacity and even Alzheimer's disease research. That is, there are study design factors that may appear so subtle as to not warrant mention in analysis manuscripts, but that can nevertheless affect interpretation and both internal and external validity of results. Finding and addressing these areas of heterogeneity could lead to discovery of novel disease variants. Heid et al.[1] conducted meta analyses for a SNV found to correlate with BMI and concluded that association estimates depended on the design; specifically, whether subjects were recruited from a general population or a healthy population. In the case of AD, our investigations strongly suggest that whether studies have home study visit capacity can likewise have a critical impact on genetic findings of associations with LOAD risk. We suspect there may be other apparently subtle design factors that may lead to important heterogeneity across studies and that may interfere with our ability to identify associations with LOAD. We could speculate that there may be important differences between people who opt for in-home visits and people willing to attend a research clinic visit, and that these differences may be related to genetic factors and predisposition to LOAD. This is consistent with the results that some SNV effects appear more extreme in the "clinic only" sample due to the induced bias while others are only significant when using all data possibly solely due to increased sample size. An important question is whether the effect estimates in the clinic only sample are correct for those individuals, or biased even for that subsample. In other words, are the differences we observe due to true effect heterogeneity or due to a bias from the selection process? Either explanation is consistent with our statistical findings.

Our findings should be considered in the context of limitations. There is some attrition in the ACT study despite including capacity for home visits. Our completeness of follow-up index[21] is exemplary but not perfect. It is not known whether similar healthy participant bias may influence the results that we observe. We doubt that would be the case due to the overall low rates of study attrition, but cannot rule out that possibility. Unmeasured and residual confounding are always possibilities in observational studies, though our findings were essentially the same with sparser "genetic style" analyses as with covariate-rich "epidemiology style" analyses. Underrepresented ethnic diversity of the ACT study is somewhat restricted, though the demographic makeup of our study cohort reflects that of King County. As in the Lambert et al. paper the analyses presented here were limited to 
people of European ancestry. Home visit capacity has been specifically advocated as a means of increasing underrepresented ethnic participation in dementia studies[22].

Our findings support the importance of home visit data collection capacity in the design of genetic studies of LOAD risk. Studies that only include research clinic study visit data collection may lead to biased conclusions. These results suggest that new LOAD genetics studies at the design stage should consider incorporating home visit data collection capacity despite its expense and administrative complexity. Existing studies should routinely report on this important detail of study design in reports of study findings. Studies that lack home study visit capacity should specify that as a possible limitation, and should be cautious in interpreting study findings. Similarly, meta-analyses of LOAD genetic analyses should consider whether home study visit capacity may be a source of between-study heterogeneity.

\section{Supplementary Material}

Refer to Web version on PubMed Central for supplementary material.

\section{Acknowledgments}

Study funding for the ACT study was from AG-06781 (Multiple PIs: E Larson and P Crane). Meredith Pfanschmidt, Sheila O'Connell, Patti Boorkman, Lisa Millspaugh, and Tiffani Rivara have conducted home-based research study visits. Some analyses were funded by R01 AG 042437 (P Crane, PI), P50 AG05136 (LEG) and K25 AG043546 (DWF).

\section{References}

1. Heid IM, Huth C, Loos RJ, Kronenberg F, Adamkova V, Anand SS, et al. Meta-analysis of the INSIG2 association with obesity including 74,345 individuals: does heterogeneity of estimates relate to study design? PLoS Genet. 2009; 5:e1000694. [PubMed: 19851442]

2. McArdle PF, Whitcomb BW. Improper adjustment for baseline in genetic association studies of change in phenotype. Hum Hered. 2009; 67:176-82. [PubMed: 19077436]

3. VanderWeele TJ, Asomaning K, Tchetgen Tchetgen EJ, Han Y, Spitz MR, Shete S, et al. Genetic variants on 15q25.1, smoking, and lung cancer: an assessment of mediation and interaction. Am J Epidemiol. 2012; 175:1013-20. [PubMed: 22306564]

4. Vanderweele TJ, Ko YA, Mukherjee B. Environmental confounding in gene-environment interaction studies. Am J Epidemiol. 2013; 178:144-52. [PubMed: 23821317]

5. Tchetgen Tchetgen E. Robust discovery of genetic associations incorporating gene-environment interaction and independence. Epidemiology. 2011; 22:262-72. [PubMed: 21228701]

6. van der Sluis S, Verhage M, Posthuma D, Dolan CV. Phenotypic complexity, measurement bias, and poor phenotypic resolution contribute to the missing heritability problem in genetic association studies. PLoS One. 2010; 5:e13929. [PubMed: 21085666]

7. Crane PK, Gibbons LE, McCurry SM, McCormick W, Bowen JD, Sonnen J, et al. Importance of home study visit capacity in dementia studies. Alzheimers Dement. 2016; 12:419-26. [PubMed: 26602628]

8. Tsuang D, Kukull W, Sheppard L, Barnhart RL, Peskind E, Edland SD, et al. Impact of sample selection on APOE epsilon 4 allele frequency: a comparison of two Alzheimer's disease samples. J Am Geriatr Soc. 1996; 44:704-7. [PubMed: 8642164]

9. Tsuang D, Larson EB, Bowen J, McCormick W, Teri L, Nochlin D, et al. The utility of apolipoprotein E genotyping in the diagnosis of Alzheimer disease in a community-based case series. Arch Neurol. 1999; 56:1489-95. [PubMed: 10593304] 
10. Lambert JC, Ibrahim-Verbaas CA, Harold D, Naj AC, Sims R, Bellenguez C, et al. Meta-analysis of 74,046 individuals identifies 11 new susceptibility loci for Alzheimer's disease. Nat Genet. 2013; 45:1452-8. [PubMed: 24162737]

11. Kukull WA, Higdon R, Bowen JD, McCormick WC, Teri L, Schellenberg GD, et al. Dementia and Alzheimer disease incidence: a prospective cohort study. Arch Neurol. 2002; 59:1737-46. [PubMed: 12433261]

12. Larson EB, Wang L, Bowen JD, McCormick WC, Teri L, Crane P, et al. Exercise is associated with reduced risk for incident dementia among persons 65 years of age and older. Ann Intern Med. 2006; 144:73-81. [PubMed: 16418406]

13. Crane PK, Walker R, Hubbard RA, Li G, Nathan DM, Zheng H, et al. Glucose levels and risk of dementia. N Engl J Med. 2013; 369:540-8. [PubMed: 23924004]

14. Teng EL, Hasegawa K, Homma A, Imai Y, Larson E, Graves A, et al. The Cognitive Abilities Screening Instrument (CASI): a practical test for cross-cultural epidemiological studies of dementia. Int Psychogeriatr. 1994; 6:45-58. discussion 62. [PubMed: 8054493]

15. American Psychiatric Association. Diagnostic and statistical manual of mental disorders. 4th. Washington, DC: American Psychiatric Association; 1994.

16. McKhann G, Drachman D, Folstein M, Katzman R, Price D, Stadlan EM. Clinical diagnosis of Alzheimer's disease: report of the NINCDS-ADRDA Work Group under the auspices of Department of Health and Human Services Task Force on Alzheimer's Disease. Neurology. 1984; 34:939-44. [PubMed: 6610841]

17. Patterson N, Price AL, Reich D. Population Structure and Eigenanalysis. PLoS Genetics. 2006; 2:2074-93.

18. Korn EL, Graubard BI, Midthune D. Time-to-event analysis of longitudinal follow-up of a survey: choice of the time-scale. Am J Epidemiol. 1997; 145:72-80. [PubMed: 8982025]

19. Fishman PA, Goodman MJ, Hornbrook MC, Meenan RT, Bachman DJ, O'Keeffe Rosetti MC. Risk adjustment using automated ambulatory pharmacy data: the RxRisk model. Med Care. 2003; 41:84-99. [PubMed: 12544546]

20. Heraud-Bousquet V, Larsen C, Carpenter J, Desenclos JC, Le Strat Y. Practical considerations for sensitivity analysis after multiple imputation applied to epidemiological studies with incomplete data. BMC Med Res Methodol. 2012; 12:73. [PubMed: 22681630]

21. Clark TG, Altman DG, De Stavola BL. Quantification of the completeness of follow-up. Lancet. 2002; 359:1309-10. [PubMed: 11965278]

22. Wrobel AJ, Shapiro NE. Conducting research with urban elders: issues of recruitment, data collection, and home visits. Alzheimer Dis Assoc Disord. 1999; 13(Suppl 1):S34-8. [PubMed: 10369516] 


\section{Research in Context (149 words)}

\section{Systematic review}

Relevant literature was reviewed using Google Scholar and PubMed. Publications that address possible bias in genetic association studies and studies without home visit capacity were cited. None address the validity of genome-wide association study results in the context of home visit capacity.

\section{Interpretation}

Our results suggest that the capacity for home research study visits is an important factor in the design of genetic studies of Alzheimer's disease and dementia. This observation implies that there are likely important design differences in genetic studies across research areas. When studies are aggregated via meta analysis or otherwise, any inference must be carefully interpreted.

\section{Future directions}

This paper indicates that home research visit capacity should be considered at the design stage of genetic association studies. Other genetic studies of Alzheimer's disease, dementia and other disorders can result in bias due to study design differences when aggregating information across potentially disparate cohorts. 


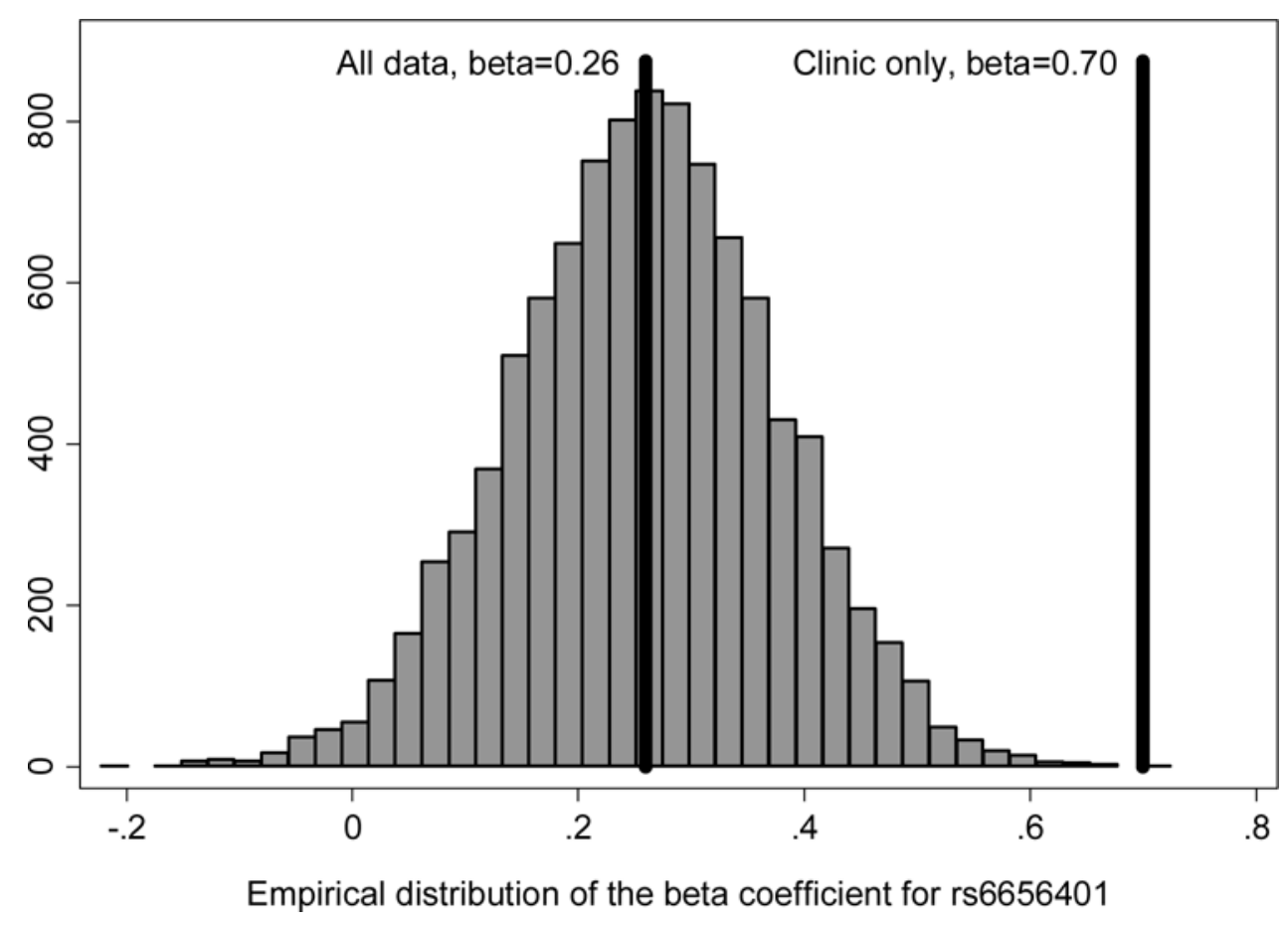

Figure 1.

Empirical distribution of the beta coefficient for the association between rs6656401 and risk of Alzheimer's disease. We sampled with replacement from the "all visits" data set a sample of people the same size as the "clinic only" data set and performed Cox regression on that data set of the association between the risk factor and risk of Alzheimer's disease. We captured the beta coefficients from those models; exponentiated beta coefficients from these models are hazard ratios. We repeated this procedure 10,000 times. The vertical line at 0.26 was the result we obtained with the entire "all visits" data set; this value is the natural logarithm of the estimated hazard ratio (1.29). The vertical line at 0.70 was the result we obtained with the "clinic only" data set; that value is the natural logarithm of the estimated hazard ratio (2.01). The graph shows that the bootstrapping results are normally distributed with a central tendency very close to the observed value from the entire "all visits" data set; the observed result from the "clinic only" data set is far from that value, and none of the 10,000 sampled data sets had values that extreme. These 10,000 beta coefficients were used to arrive at the $p$-value of 0.0001 shown in Table 3 


\section{Table 1}

Demographic and clinical characteristics $(n=1697)$.

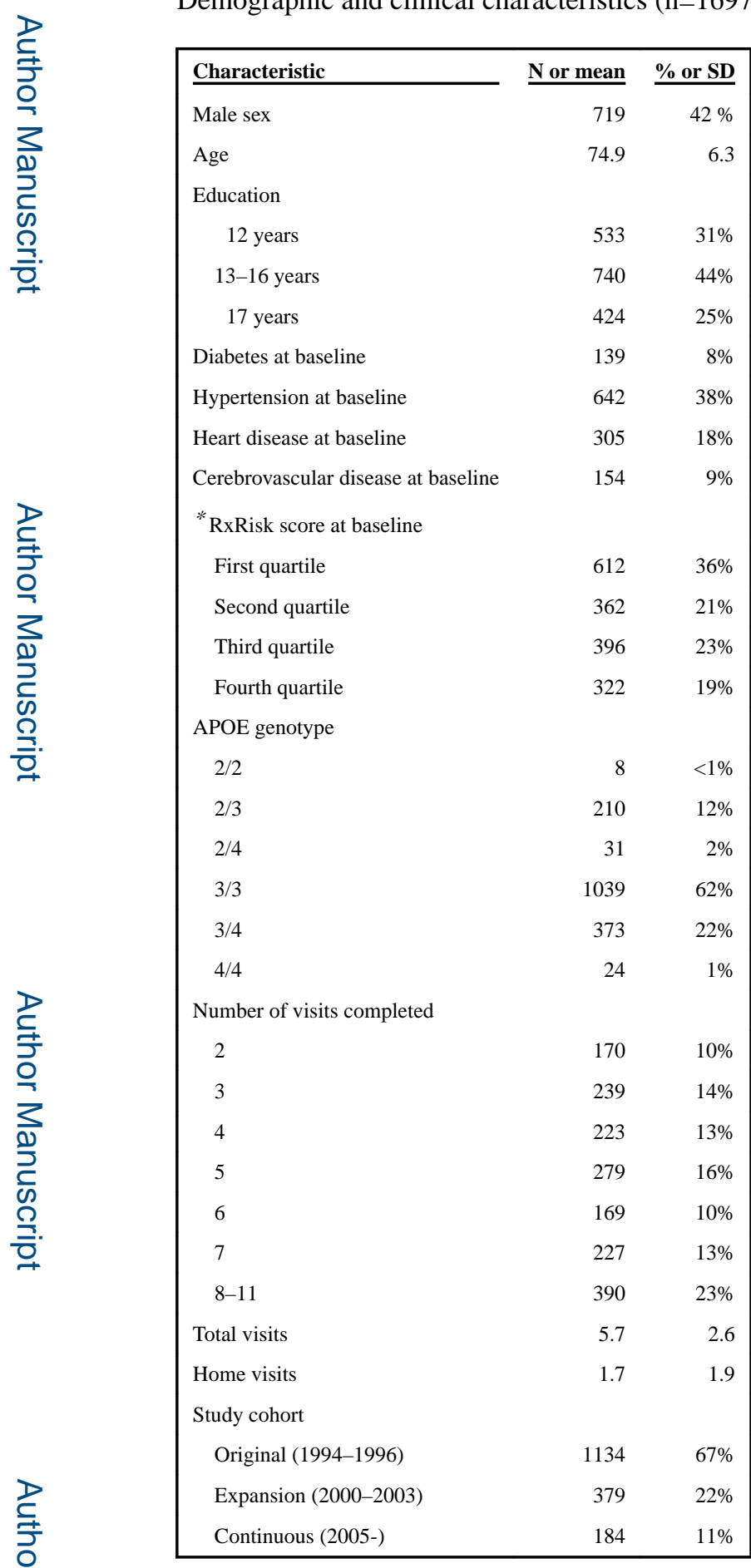

* Higher RxRisk scores represent greater comorbidity[19]

Alzheimers Dement. Author manuscript; available in PMC 2018 August 01. 


\section{Table 2}

\section{Distribution of visit patterns}

\begin{tabular}{lc}
\hline Pattern & $\mathbf{N}(\%)$ \\
\hline All visits occurred at the clinic & $621(37 \%)$ \\
One or more clinic visits followed by one or more home visits & $845(50 \%)$ \\
One or more clinic visits followed by one or more home visits and at least one more clinic visit & $34(2 \%)$ \\
One or more home visits followed by one or more clinic visits & $11(1 \%)$ \\
One or more home visits followed by one or more of clinic visits and at least one more home visit & $23(1 \%)$ \\
All visits occurred at home & $163(10 \%)$ \\
\hline
\end{tabular}


Fardo et al.

Page 15

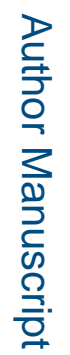

D

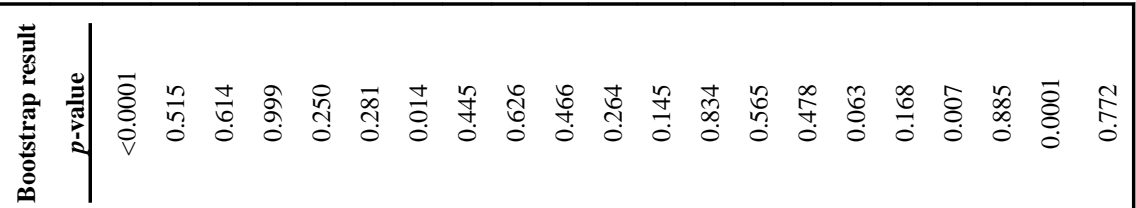

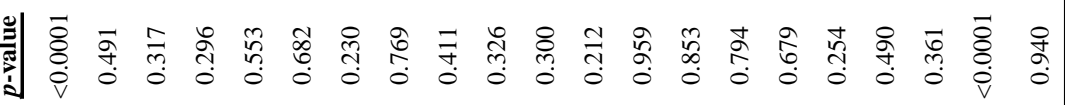

竞

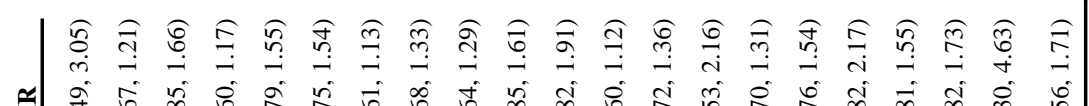

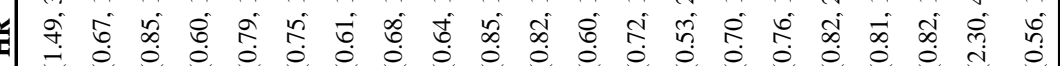

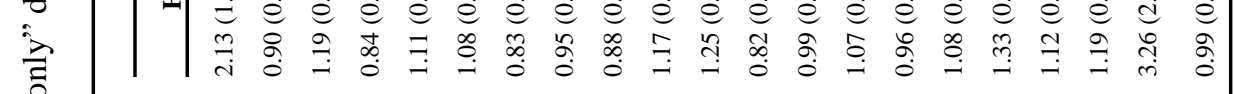

:

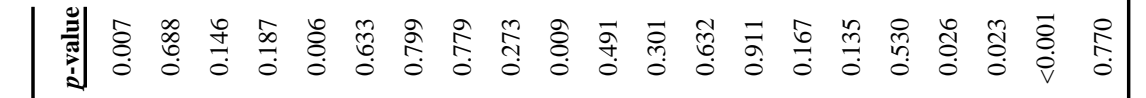

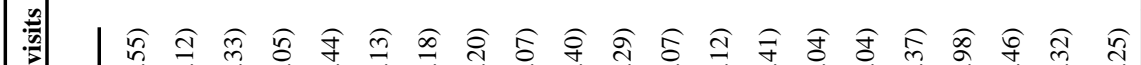

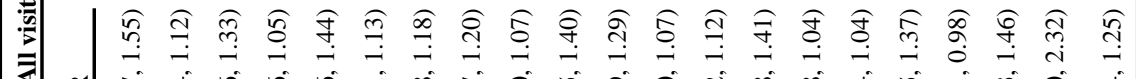

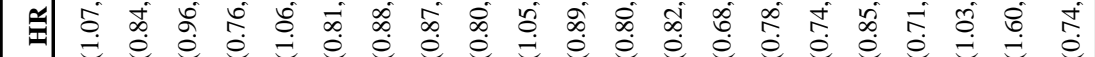

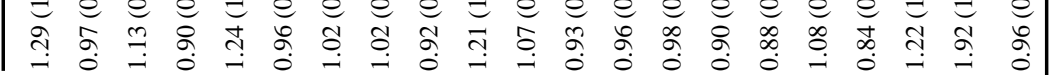

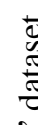

ग

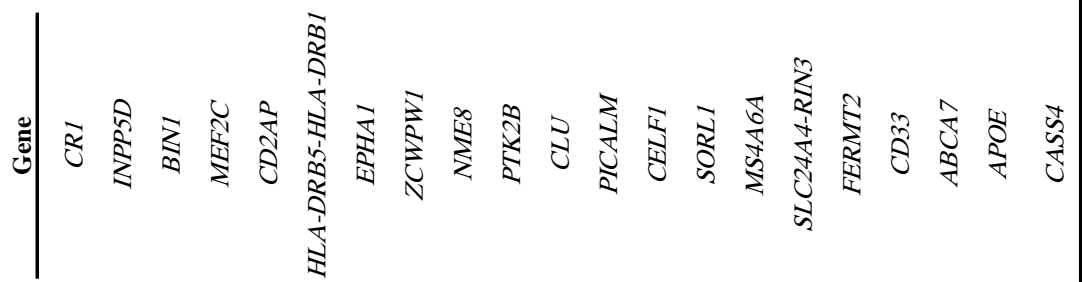

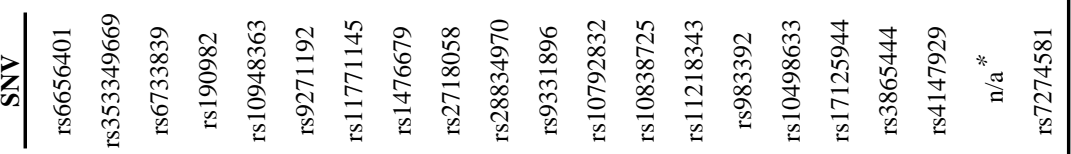

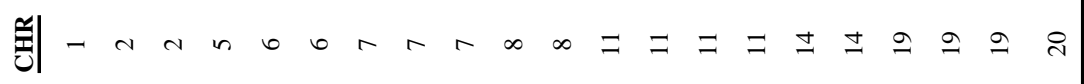

